\title{
The Classification of Quasi-Regular Polyhedra of Genus 2
}

\author{
Reinhard Franz and Daniel Huson \\ Fakultät für Mathematik, Universität Bielefeld, Universitätsstraße, \\ W-4800 Bielefeld 1, Federal Republic of Germany
}

\begin{abstract}
The method of chamber systems is used to provide a complete list of all possible tessellations of the closed, orientable surface of genus 2 by (topological) $n$-gons and $m$-gons $(n, m>2)$ satisfying a certain local symmetry condition. Using a computer program it is shown that (up to homeomorphism) there are precisely 379 such quasi-regular polyhedra. S. Bilinski constructed the first one for each of the 17 possible combinations of $m$ - and $n$-gons using geometrical methods. It is the intention of the authors to demonstrate the usefulness and suitability of chamber systems in dealing with problems of the above type.
\end{abstract}

\section{Quasi-Regular Polyhedra}

A (topological) polyhedron $\mathscr{P}$ (of genus $p$ ) is a compact, orientable 2-manifold (in $\mathbb{E}^{3}$ ) of genus $p$ divided into simply connected open regions by a finite number of arcs (and simple closed curves) called edges. Such a region, together with its boundary, is called a face. Edges meet only at their endpoints called vertices and each vertex is incident to at least three edges (where loops are counted twice) (see [16]).

As in [2], a (topological) polyhedron $\mathscr{P}$ is called (locally) quasi-regular if each of its vertex-cycles has the form

$$
\underset{1}{(m, n, m, n, \ldots, m, n)}
$$

with $n>m>2$ and $s>1$. In other words, when "going around" a vertex, we alternately encounter $m$-edged and $n$-edged faces (exactly $s$ of each). We call $\mathscr{P}$ globally quasi-regular if its automorphism group acts transitively on its set of edges.

There is a simple construction of globally quasi-regular polyhedra which we would like to mention: Start with a regular map $\mathscr{M}$ of type $\{n, m\}$. Truncate $\mathscr{M}$; 
that is, "cut off" the vertices of $\mathscr{M}$ up to the midpoints of the edges; or equivalently, join up the midpoints of the edges of $\mathscr{M}$. This gives a globally quasi-regular polyhedron with parameters $n$ and $m$ and $s=2$. For genus $g=2$ this gives several polyhedra for free (see p. 140 of [4]).

For a quasi-regular polyhedron $\mathscr{P}$ of genus $p$, with $\alpha_{0}$ vertices, $\alpha_{1}$ edges, and $\alpha_{2}$ faces $\left(q_{m}\right.$ of which have $m$ edges and $q_{n}$ of which have $n$ edges), the following system of Diophantine equations holds:

$$
\begin{aligned}
s \alpha_{0} & =m q_{m}=n q_{n}=\alpha_{1}, \\
\alpha_{2} & =q_{m}+q_{n},
\end{aligned}
$$

and

$$
2(1-p)=\alpha_{0}-\alpha_{1}+\alpha_{2} \quad \text { (Euler formula) }
$$

with $m, q_{m}, n, q_{n}$, and $s$ as above. Note that the numbers $\alpha_{0}, \alpha_{1}$, and $\alpha_{2}$ are completely determined by the parameters $m, q_{m}, n, q_{n}$, and $s$.

For $p=2$ there are exactly 17 choices of positive integers $m, q_{m}, n, q_{n}$, and $s$ that satisfy (2). In [2] and [3] Bilinski shows that, for each of these 17 solutions, a corresponding polyhedron $\mathscr{P}$ exists. In [9] quasi-regular polyhedra are discussed in terms of their associated "chamber systems." There it is shown, in the case $q_{m}=4, m=3, q_{n}=3, n=4, s=4$, how chamber systems can be used to determine all the corresponding homeomorphism classes of (oriented) quasiregular polyhedra. ${ }^{1}$

This paper describes an algorithm based on the chamber-system approach. By producing all appropriate chamber systems, we obtain the new result that there exist precisely 379 classes of quasi-regular polyhedra of genus 2 . Exactly ten of these classes consist of globally quasi-regular polyhedra. Representatives for the latter are displayed in Figure 4.

\section{The Chamber System of a Polyhedron}

To define the chamber system of a polyhedron $\mathscr{P}$ of genus $p$, let us consider a barycentric subdivision of $\mathscr{P}$ (see Fig. 1). Such a triangulation $\mathscr{P}^{\prime}$ of $\mathscr{P}$ can be obtained by first adding to the interior of each edge and each face of $\mathscr{P}$ exactly one new vertex and then, for every face, connecting, via new edges, the vertex placed in that face to all the vertices contained in the boundary of that face. Thus

\footnotetext{
Though not explicitly mentioned there, the ten systems $(\mathscr{D} ; \alpha, \beta)$ listed in [9] represent all homeomorphism classes of oriented quasi-regular polyhedra of the type considered there. Six of the ten oriented polyhedra are homeomorphic to their "mirror image" (by an orientation-preserving homeomorphism!), the polyhedra $2 a 2 b 2 c-1,2 a 2 b 2 c-2$ and the polyhedra $2 b 2 b 2 d-2,2 b 2 b 2 d-3$ are mirror images of each other. Hence, there are precisely eight homeomorphism classes of such polyhdedra if orientation is neglected.
} 


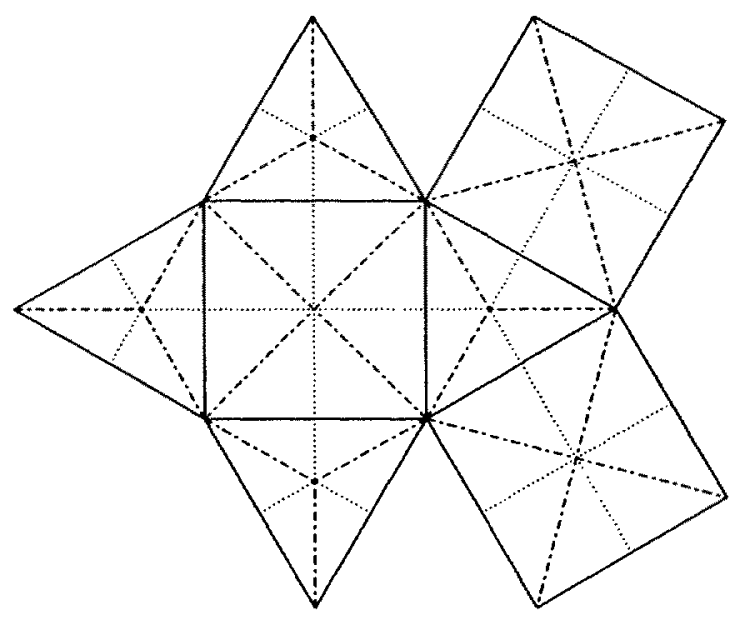

Fig. 1. The barycentric subdivision of a section of a typical polyhedron $\mathscr{P}$. The edges of $\mathscr{P}$ are shown as unbroken lines whereas dotted and dashed lines indicate the new edges added to $\mathscr{P}$.

$\mathscr{P}^{\prime}$ has three types of vertices and we call a vertex of $\mathscr{P}^{\prime}$ either a $0-, 1-$, or 2 -vertex depending on whether it lies in (the interior of) a vertex, an edge, or a face of $P$. Note that every face of $\mathscr{P}^{\prime}$ possesses exactly one vertex of each of the three types.

The intersection of two different faces of $\mathscr{P}^{\prime}$ is either empty, a vertex, or it consists of exactly one edge connecting two vertices of different types. By the color of an edge $e$ of $\mathscr{P}^{\prime}$ we mean the number $k$ with the property that $e$ contains a $i$-vertex and a $j$-vertex but not a $k$-vertex, where $\{i, j, k\}=\{0,1,2\}$.

Now the chamber system $G(\mathscr{P})=(\mathscr{X}, \mathscr{U})$ of $\mathscr{P}$ is defined as the dual of the graph given by the 1-skeleton of $\mathscr{P}^{\prime}$. That is, the vertex set $\mathscr{X}$ of $G$ consists of the faces of $\mathscr{P}^{\prime}$ called chambers and two different vertices $x, y \in \mathscr{X}$ are connected in $G$ by an edge of color $k$ (a $k$-edge) in $\mathscr{U}$ if and only if, as faces of $\mathscr{P}^{\prime}$, they intersect in an edge of color $k$. Note that different barycentric subdivisions of $\mathscr{P}$ yield isomorphic chamber systems.

It follows from our construction that each vertex of the chamber system of a polyhedron $\mathscr{P}$ is incident to exactly one $i$-edge (for all $i \in\{0,1,2\}$ ). Furthermore the graph does not contain any loops (since, as a 2 -manifold, $\mathscr{P}$ has no boundary), is bipartite (since $\mathscr{P}$ is orientable), and has genus $p$. Removing all the edges of color $k(k \in\{0,1,2\})$ yields a spanning (planar) subgraph whose connected components (called $i$-j-components) are cycles consisting of alternating $i$ - and $j$-edges $(\{i, j, k\}=\{0,1,2\})$, called $i$-j-cycles. Note that there is a canonical one-to-one correspondence between the 0-1-, 0-2-, and 1-2-cycles and the faces, edges, and vertices of $\mathscr{P}$, respectively. Furthermore, we have the following. theorem which allows a classification of quasi-regular polyhedra in terms of their associated chamber systems (see [9]):

Theorem A. There exists a one-to-one correspondence between the homeomorphism classes of quasi-regular polyhedra of genus $p$ as defined above and the isomorphism 
classes of finite, connected, edge-colored, bipartite graphs

$$
G=(\mathscr{X}, \mathscr{U} \subset\{\{a, b\} \mid a, b \in \mathscr{X}\} \times\{0,1,2\})
$$

satisfying the following conditions:

(P1) There exist natural numbers $m, n$ with $2<m<n$ such that $G$ consists of exactly $q_{m} 0-1-c y c l e s$ of cardinality $2 m$ and $q_{n} 0-1-c y c l e s$ of cardinality $2 n$.

(P2) Every 0-2-cycle is of cardinality 4.

(P3) All 1-2-cycles have the same cardinality, say 4s, which is larger than 4.

(P4) Every 0-2-cycle intersects exactly one 0-1-cycle of cardinality $2 n$ and one of cardinality $2 \mathrm{~m}$.

(P5) The parameters $q_{m}, m, q_{n}, n$, and s satisfy the equation

$$
2(1-p)=\frac{m q_{m}}{s}-m q_{m}+q_{m}+q_{n}
$$

Remark. Since the numerical invariants $m, q_{m}, n, q_{n}$, and $s$ of the quasi-regular polyhedron $\mathscr{P}$ coincide with the numerical invariants of the associated chamber system $G(\mathscr{P})$ defined in (P1), (P2), and (P3) they are denoted by the same symbols.

\section{Algorithm POLYHEDRA}

Theorem A shows that the two following problems can be translated into each other:

(a) Producing all homeomorphism classes of quasi-regular polyhedra of genus $p$.

(b) Enumerating all isomorphism classes of connected arc-colored bipartite graphs $G=(\mathscr{X}, \mathscr{U} \subset\{\{a, b\} \mid a, b \in \mathscr{X}\} \times\{0,1,2\})$ satisfying conditions (P1)-(P5).

In this paper we formulate a simple algorithm which generates "standard representatives" for all isomorphism classes of chamber systems corresponding to a given set of parameters $m, q_{m}, n, q_{n}, s(n>m>2, s>1)$, and $p$ satisfying (2). We start with a simple version of the algorithm based on the "brute-force" method of generating permutations (see [19]), which is too slow to produce complete results. Then we discuss how to speed up the algorithm.

Picture Algorithm POLYHEDRA operating on a single graph $G=(\mathscr{X}, \mathscr{U})$, successively adding and deleting edges in that graph, and, whenever appropriate, producing output by "printing" a copy of the graph in its current state.

Before the algorithm is applied, graph $G$ is initialized as follows. Give graph $G$ exactly $N=4 q_{m} m=4 q_{n} n$ vertices which, for the sake of simplicity, are identified with the numbers $1, \ldots, N$, thus $\mathscr{X}=\{1, \ldots, N\}$. Set $\mathscr{A}:=\{1, \ldots, N / 2\}$ and $\mathscr{B}:=$ $\{N / 2+1, \ldots, N\}$. Next we define the 0 - and 1-edges in $\mathscr{U}$ (note that our algorithm will leave these edges fixed): Any two vertices $i$ and $i+1$ are to be connected by a 0 -edge if $i$ is odd. Then 1-edges are used to create $q_{m}$ consecutive 0-1-cycles of cardinality $2 m$, and $q_{n}$ such cycles of cardinality $2 n$ in $G$ (property (P1)!). Figure 

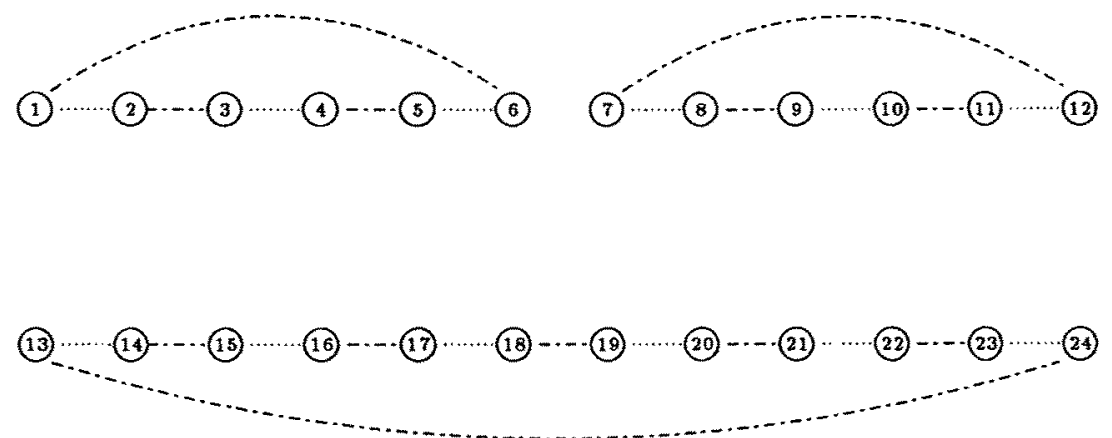

Fig. 2. The initial state of a typical graph $G$. Vertices are indicated by small circles and $0-$ (or 1-) edges are drawn as dotted (or dashed) lines.

2 indicates exactly how this is to be done. Note that the vertex set of $\mathscr{A}$ (or $\mathscr{B}$ ) induces a graph consisting of $q_{m}$ (or $q_{n}$ ) consecutive 0-1-cycles of cardinality $m$ (or $n$, respectively).

Graph $G$ is now ready to have the algorithm applied to it. Before proceeding to define the algorithm, we briefly introduce some more notation. To 2-connect two vertices $i$ and $j$ means to connect them by a 2-edge. A vertex $i$ that is not 2-connected to any other vertex is called free. Note that to help ensure property (P4) the algorithm may only 2-connect two vertices $i$ and $j, i<j$, if $i \in \mathscr{A}$ and $j \in \mathscr{B}$.

Because we are only interested in bipartite graphs, we can always assume that two 2-connected vertices $i$ and $j$ are either both even or both odd. Furthermore, if, while running the algorithm, we want to 2 -connect an odd vertex $i \in \mathscr{A}$ to an odd vertex $j \in \mathscr{B}$, then to ensure property (P2) the vertices $i+1$ and $j+1$ must also be 2-connected. An ordered pair $(i, i+1)$ of vertices (in $\mathscr{A}$ or $\mathscr{B}$ ) is called a 0 -pair (in $\mathscr{A}$ or $\mathscr{B}$, respectively) if they are joined by a 0 -edge, which is the case whenever $i$ is odd. We 2-connect two pairs $(i, i+1)$ and $(j, j+1)$ by 2 -connecting $i$ to $j$ and $i+1$ to $j+1$. A 0 -pair of free vertices is called free. Note that initially all 0 -pairs in $G$ are free. Finally, a 0 -pair $(i, i+1)$ is called a head if $i$ is the smallest vertex for some 0-1-cycle.

\section{Algorithm POLYHEDRA}

Input: Initially (i.e., at recursion level 0 ) graph $G$ as defined above, all vertices free. At recursion level $t$ : Graph $G$ with $2 t$ new 2-edges added.

Output: One representative for each isomorphism class of connected, bipartite graphs that satisfy (P1)-(P5).

if $\mathscr{A}$ contains a free 0 -pair then \{

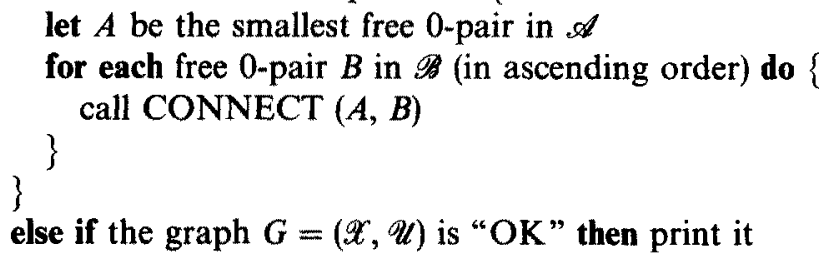




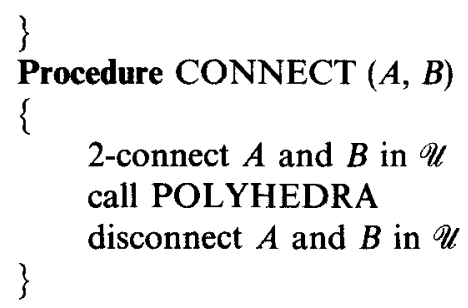

In the else if line graph $G$ is "OK" if it has the following properties:

(OK1) Every 0-pair has been 2-connected.

(OK2) Every 1-2-cycle is of cardinality 4s.

(OK3) The graph is not isomorphic to any graph previously printed out by the algorithm.

\section{Speeding up the Algorithm}

While "running" the recursive algorithm POLYHEDRA let us indicate the current "level of recursion" by a subscript $t$. So initially Algorithm POLYHEDRA $A_{0}$ is called and for the $t$ th nested call of the algorithm we write POLYHEDRA . $_{t}$

Now assume that Algorithm POLYHEDRA is running and that it has already 2-connected the first $t<N / 4$ of the 0 -pairs in $\mathscr{A}$ and that it has just entered POLYHEDRA $_{t}$ (see Fig. 3). Algorithm POLYHEDRA takes the smallest free 0 -pair $A$ in $\mathscr{A}$ and 2-connects it to the smallest free 0-pair $B$ in $\mathscr{B}$. It then goes on to call POLYHEDRA ${ }_{t+1}$ (which may or may not lead to some graphs being printed out). Once this call has been completed, the 0-pairs $A$ and $B$ are disconnected. Algorithm POLYHEDRA ${ }_{t}$ would now go on to select the next free 0 -pair $B^{\prime}>B$ in $\mathscr{B}$ and then to reenter the main loop to 2-connect $A$ and $B^{\prime}$, etc. However, if $A$ is a head, then repeating the main loop will not produce any graphs not isomorphic to ones already printed during the first execution of the main loop. The reason for this is that, with respect to isomorphism, the 0-pair $A$ and all

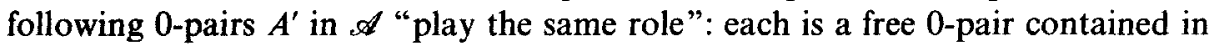

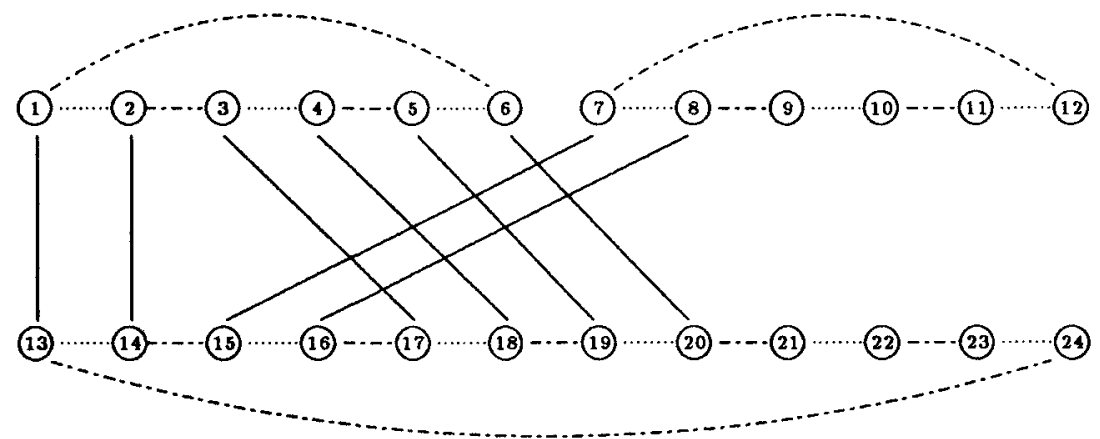

Fig. 3. An example of the typical state of a graph $G$ as the algorithm enters POLYHEDRA, In this case $t=4$ and the algorithm is just about to 2-connect the 0-pair $(9,10)$. Vertices are indicated by small circles and 0-(1- or 2-) edges are drawn as lines that are dotted (dashed or unbroken, respectively). 
a 0-1-cycle of cardinality $2 m$, which consists entirely of free 0-pairs. Hence, if we were to 2-connect $A$ to $B^{\prime}$, then in POLYHEDRA $A_{t+1}$ some $A^{\prime}>A$ would be 2-connected to $B$ and we would not obtain a new isomorphism type of graph. Now consider Algorithm POLYHEDRA, under the assumption that $A$ is not a head. If at some point the main loop chooses a free 0 -pair $B^{\prime}$ in $\mathscr{B}$ that is a head, then the above argument applies similarly to $B^{\prime}$ and again, after performing POLYHEDRA $_{t+1}$ with $A$ and $B^{\prime}$ 2-connected, Algorithm POLYHEDRA $A_{t}$ can be aborted.

So we have seen that it suffices if our algorithm considers only graphs $G$ that have the following two properties for any pair of 0-pairs $A$ and $A^{\prime}$ in $\mathscr{A}$ 2-connected to 0 -pairs called $B$ and $B^{\prime}$ in $\mathscr{B}$ :

(a) If $A$ is a head and $A \leq A^{\prime}$, then $B \leq B^{\prime}$.

(b) If $B$ is a head and $B \leq B^{\prime}$, then $A \leq A^{\prime}$.

We can ensure that only such graphs are considered by adding the following line to the bottom of the main loop:

\section{if $A$ is a head or $B$ is a head then end}

where the statement end means end the "current incarnation" of POLYHEDRA (but not the whole algorithm).

We take a further step to speed up the algorithm. It consists of making the procedure CONNECT ensure that, from the beginning, the algorithm only considers and produces graphs whose 1-2-cycles have the correct cardinality. For any intermediate graph produced by the algorithm, let $\mathscr{C}(i)$ denote the 1-2component that contains the vertex $i$. Any such set $\mathscr{C}(i)$ is either a 1-2-cycle, or a chain using alternating 1- and 2-edges (a 1-2-chain). Let $i \in \mathscr{A}$ and $j \in \mathscr{B}$ be two vertices not incident to a 2 -edge. Then $\mathscr{C}(i)$ and $\mathscr{C}(j)$ are 1-2-chains. If $\mathscr{C}(i) \cap \mathscr{C}(j) \neq$ $\varnothing$, then $\mathscr{C}(i)=\mathscr{C}(j)$ and 2 -connecting $i$ and $j$ produces a 1-2-cycle of cardinality $\# \mathscr{C}(i)$. Otherwise, 2-connecting $i$ and $j$ produces a 1-2-chain of cardinality $\# \mathscr{C}(i)+\# \mathscr{C}(j)$.

Replace the entire procedure CONNECT by this new version:

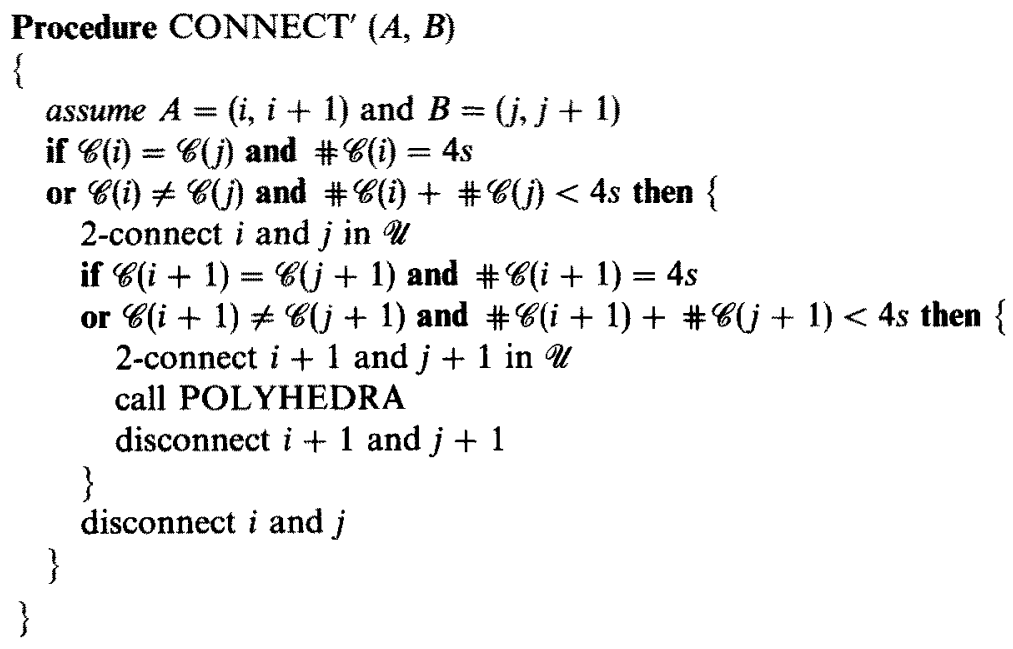


Finally, it follows from properties (a) and (b) that adding the lines

if $A$ is not the smallest 0 -pair in $\mathscr{A}$ then

if $A$ is a head and the smallest free 0 -pair in $\mathscr{B}$ is a head then end

to POLYHEDRA directly under the line marked (*) ensures that the graphs considered by the algorithm are connected.

After making all the the above modifications to the algorithm, in the else if line only the property (OK3) has to be checked, (OK1) and (OK2) are necessarily satisfied. Here is the modified algorithm:

\section{Algorithm POLYHEDRA'}

Input: Initially (i.e., at recursion level 0) graph $G$ as defined above, all vertices free. At recursion level $t$ : graph $G$ with $2 t$ new 2-edges added.

Output: One representative for each isomorphism class of connected, bipartite graphs that satisfy (P1)-(P5).

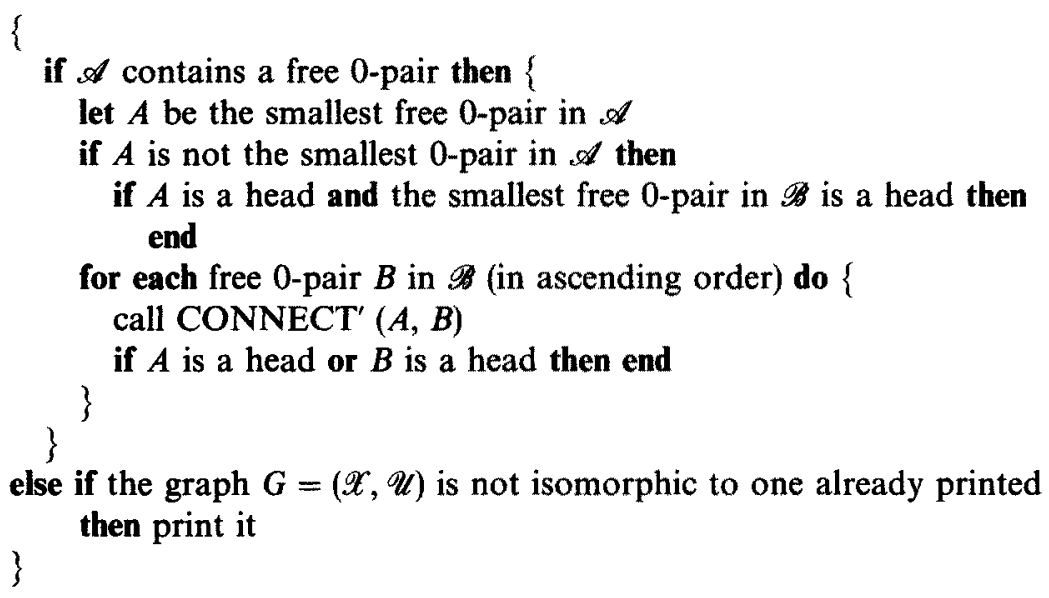

\section{Results and Comments}

Implementing the final version of Algorithm POLYHEDRA on a computer yields the following results:

Theorem B. There are exactly 379 homeomorphism classes of quasi-regular polyhedra of genus 2, ten of which are globally quasi-regular and 225 of which have orientation-reversing automorphisms (see Table 1).

Figure 4 displays a representative of each of the ten classes of globally quasiregular polyhedra of genus 2 .

A remark on the computation time: our implementation of Algorithm POLYHEDRA' needed approximately 4000 minutes for the computation of the 90 classes 

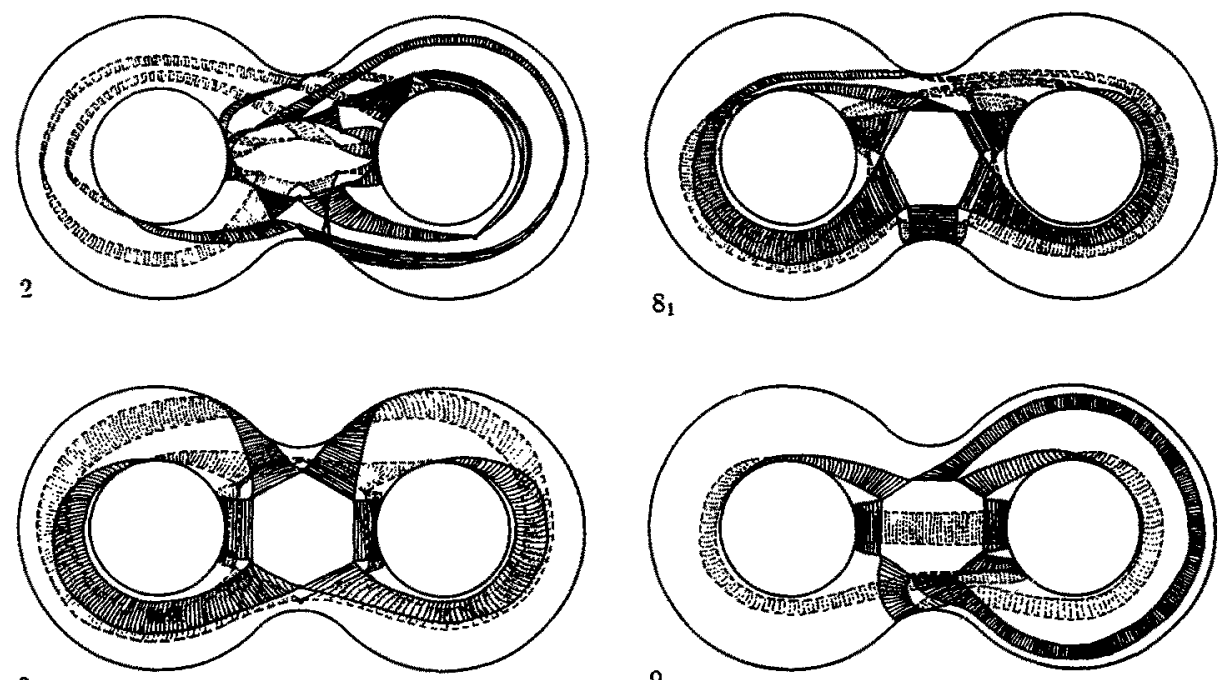

$8_{2}$
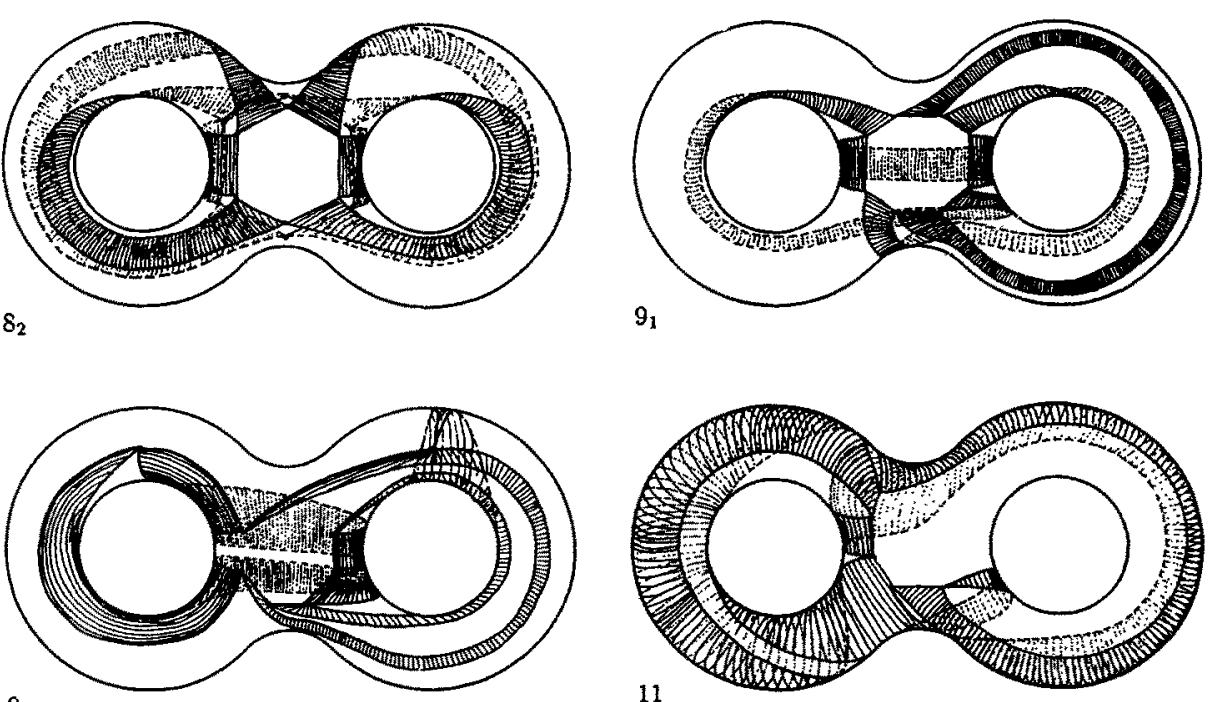

$9_{2}$
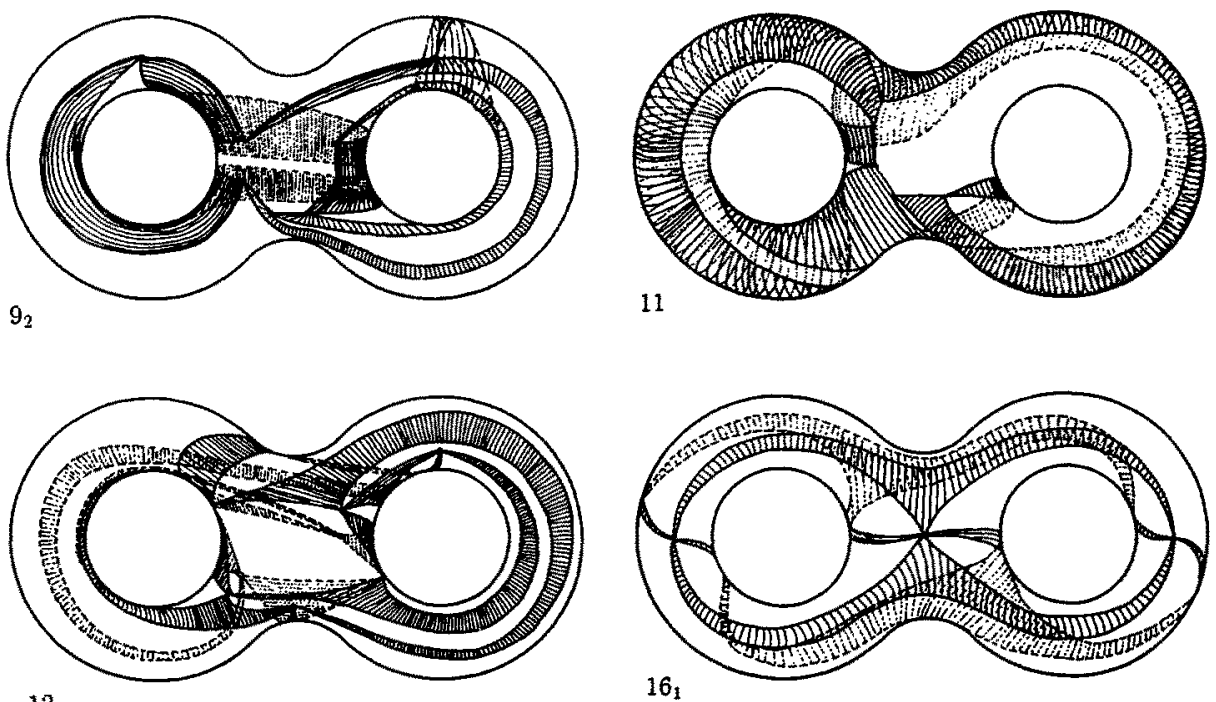

12

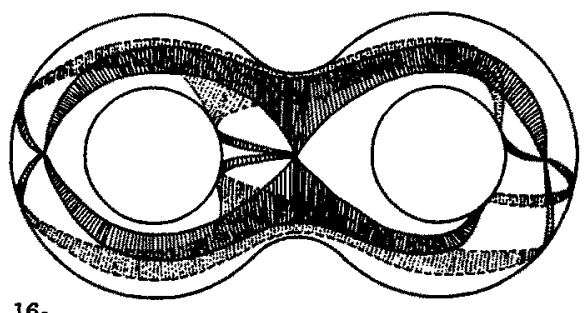

162

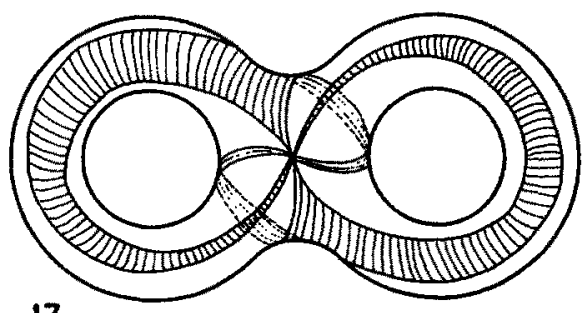

17

Fig. 4. Representatives of all ten classes of globally quasi-regular polyhedra of genus 2. 
Table 1. Distribution of the 379 classes of quasi-regular polyhedra.*

\begin{tabular}{rrrrrrrrrr}
\hline Case & $q_{m}$ & $m$ & $q_{n}$ & \multicolumn{1}{c}{$n$} & $s$ & \multicolumn{1}{c}{$N$} & $Q$ & $Q^{+}$ & $G$ \\
\hline 1 & 28 & 3 & 12 & 7 & 2 & 336 & 90 & 155 & 0 \\
2 & 16 & 3 & 6 & 8 & 2 & 192 & 57 & 77 & 1 \\
3 & 12 & 3 & 4 & 9 & 2 & 144 & 24 & 37 & 0 \\
4 & 10 & 3 & 3 & 10 & 2 & 120 & 15 & 20 & 0 \\
5 & 8 & 3 & 2 & 12 & 2 & 96 & 20 & 25 & 0 \\
6 & 6 & 3 & 1 & 18 & 2 & 72 & 8 & 9 & 0 \\
7 & 10 & 4 & 8 & 5 & 2 & 160 & 51 & 75 & 0 \\
8 & 6 & 4 & 4 & 6 & 2 & 96 & 33 & 40 & 2 \\
9 & 4 & 4 & 2 & 8 & 2 & 64 & 18 & 19 & 2 \\
10 & 3 & 4 & 1 & 12 & 2 & 48 & 6 & 6 & 0 \\
11 & 2 & 5 & 1 & 10 & 2 & 40 & 6 & 7 & 1 \\
12 & 8 & 3 & 6 & 4 & 3 & 96 & 21 & 28 & 1 \\
13 & 5 & 3 & 3 & 5 & 3 & 60 & 7 & 9 & 0 \\
14 & 4 & 3 & 2 & 6 & 3 & 48 & 7 & 8 & 0 \\
15 & 3 & 3 & 1 & 9 & 3 & 24 & 4 & 4 & 0 \\
16 & 4 & 3 & 3 & 4 & 4 & 48 & 8 & 10 & 2 \\
17 & 2 & 3 & 1 & 6 & 6 & 24 & 4 & 4 & 1 \\
\hline
\end{tabular}

* Columns 2-6 contain the 17 solutions of the system of Diophantine equations (2) for $p=2, N$ is the cardinality of the vertex sets, $Q$ is the number of homeomorphism classes of the corresponding quasi-regular polyhedra, $Q^{+}$is the number of homeomorphism classes of the corresponding oriented quasi-regular polyhedra, and $G$ is the number of globally quasiregular polyhedra.

of quasi-regular polyhedra in Case 1, where the associated chamber systems have $N=336$ vertices.

Modified versions of Algorithm POLYHEDRA are presently being used to solve classification problems concerning more general polyhedra. Similar algorithms based on the theory of "Delaney symbols" developed by Dress and others (see, e.g., [6], [8], [10], and [13]) have been used successfully to enumerate periodic tilings of the plane (see, e.g., [11], [12], and [17]). Work is presently being done on developing computer graphics programs that automatically draw the associated structure to a given Delaney symbol or chamber system. Following Tutte (see [20]) recursion formulas counting homeomorphism classes of various types of "flagged" regular polyhedra can also be developed (see [1], [7], [14], and [15]).

\section{References}

1. D. Arques, Enumération et Codage des cartes et Hypercartes plainaires pointées, Publ I.R.M.A. Actes Séminares Lotharingien $12^{\circ}$ session, Strasbourg, 1985, pp. 1-66.

2. S. Bilinski, Die quasiregulären Polyeder vom Geschlecht 2, Sitzungsber. Österr. Akad. Wiss. Math.-Natur. Kl. Abt. II 194 (1985), 63-78.

3. S. Bilinski, Die quasireguiären Polyeder zweiter Stufe, Sitzungsber. Österr. Akad. Wiss. Math.Natur. Kl. Abt. II 196 (1987), 1-12. 
4. H. S. M. Coxeter and W. O. J. Moser, Generators and Relations for Discrete Groups, 4th edition, Springer-Verlag, Berlin, 1980.

5. A. W. M. Dress, The 37 combinatorial types of regular "Heaven and Hell" patterns in the euclidean plane, M. C. Escher: AA and Science, Edited by H. S. M. Coxeter et al., Elsevier Science, (North-Holland), Amsterdam, 1986, pp. 35-44.

6. A. W. M. Dress, Regular polytopes and equivariant tessellations from a combinatorial point of view, Proc. Conf. on Algebraic Topology, Göttinger, 1984, SLN 1172, 1986, pp. 56-72.

7. A. W. M. Dress, Einige Ergebnisse (ohne Beweise) über die Anzahlen 2-dimensionaler regulärer Parkettierungen, Manuscript, Bielefeld, 1986.

8. A. W. M. Dress, Presentations of discrete groups, acting on simply connected manifolds, Adv. in Math. 63(2) (1987), 196-212.

9. A. W. M. Dress and R. Franz, Zu einer Frage von Herrn S. Bilinski, Pflasterungen von Brezelflächen betreffend, Jug. Akad. Zn. Umj. Zagreb Rad 428 (1987), 83-101.

10. A. W. M. Dress and R. Franz, A Recursive Structure for the Family of Periodic Tilings, SLN (to appear).

11. A. W. M. Dress and D. Huson, On tilings of the plane, Geom. Dedicata 24 (1987), 295-310.

12. A. W. M. Dress and D. Huson, Heaven and Hell tilings, Rev. Topologie Struct. (to appear).

13. A. W. M. Dress and R. Scharlau, Zur Klassifikation äquivarianter Pflasterungen, Mitteilungen aus dem Math. Seminar Giessen, Coxeter-Festschrift 164 (1984).

14. R. Franz, Zum rekursiven Aufbau der Familie der periodischen Pflasterungen, Ph.D. thesis, Bielefeld, 1988, pp. 31-66.

15. R. Franz, Zur Abzählung periodischer Pflasterungen der euklidischen Ebene, Publ. I.R.M.A. session $19^{e}$ Séminaire Lotharingien, Strasbourg, 1988.

16. B. Grünbaum and G. C. Shephard, Tilings and Patterns, Freeman, New York, 1987.

17. D. Huson, Die Klassifikation 2-isohedraler Pflasterungen der euklidischen Ebene, Diplomarbeit, Bielefeld, 1986.

18. D. Huson, Patches, Stripes and Net-Like Tilings, Ph.D. thesis, Bielefeld, 1989.

19. R. Sedgewick, Permutation generation methods, Comput. Surveys 9(2) (1977), 137-164.

20. W. T. Tutte, A census of planar maps, Canad. J. Math. 15 (1063), 249-271.

Received April 16, 1990, and in revised form October 25, 1990. 